\title{
The Solar Activity during the Holocene: Amplitude Variations of the Quasy-century and Quasi-two-century Solar Cycles
}

\author{
Boris Komitov ${ }^{1}$, Boncho Bonev ${ }^{2}$, Kaloyan Penev ${ }^{3}$ and Stephano Sello ${ }^{4}$ \\ ${ }^{1}$ Institute of Astronomy, Bulgarian Academy of Sciences, PO Box 39, Bulgaria, \\ 6003 Stara Zagora; komitov@mbox.digsys.bg \\ ${ }^{2}$ Laboratory for Extraterrestrial Physics, Code 690, NASA's Goddard Space Flight Center, \\ Greenbelt, MD 20771; bbonev@lepvax.gsfc.nasa.gov \\ ${ }^{3}$ Harvard University, Mail Stop 10, 60 Garden Street, Cambridge, MA 02138; \\ kpenev@cfa.harvard.edu \\ ${ }^{4}$ Mathematical and Physical Models, Enel Research, Via Andrea Pisano 120, 56122 PISA, \\ Italy; stefano.sello@enel.it
}

We summarize the recent results and prospective work in two parallel studies (Komitov; Bonev, Penev and Sello) of the long-term trends in solar variability that can be deduced from both indirect data and optical records. Analysis of data from ${ }^{14} \mathrm{C}$ measurements (Stuiver et al. 1998), aurorae (Schove series: Shove 1983), and direct astronomical records (group sunspot number) (Hoyt \& Schatten 1998) focus on the stability and changes in amplitude of the cycles with duration near one and two centuries. Although these two projects have been carried independently and different methods have been used. We present them in a joint fashion in order to emphasize the common direction of their work.

The $T-R$ periodogramm analysis for cycles detection in time series has been used in Komitov's study. The details of this method are described in a few works (Komitov 1986, Komitov 1997, Benson et al. 2003). The integral power $S$-parameters, which are derived on the basis of results from the $T$ - $R$ periodogram analysis of time series (Komitov 1999) has also been used for estimation of cycles' magnitudes. In the second study (Bonev, Penev, Sello 2004) a "moving windows" $T-R$ periodogramm method as well as a multiresolution wavelet analysis has been used.

Komitovs study points out that a 2200-2500-year cycle plays an important role in the variations of the ${ }^{14} \mathrm{C}$ production rate during the last 10000 years (Holocene). This cycle has a likely solar origin and also shows strong climatic appearences. Its minima correspond to the Maunder-type minima of solar activity.

Very important is the fact that the present structure and length of this quasybimillenial solar oscilation has been established about 7000-7500 years ago. During the early part of Holocene this cycle has been shorter than now with length 1800 years.

During the last $\sim 5000$ years a modulation of the solar bi-centurial cycle's amplitude by the $\sim 2300$-year cycle is observed. During the earlier part of Holocene $(\sim 8000-3000$ BC) this "modulating" supermillenial cycle is expressed as a quasyperiodic trend, which corresponds to a period $T \approx 3000$ years. A more detailed analysis points out that the "critical" moment of changing the modulation regime is near to $5000 \mathrm{BC}$. This moment is very interesting in climatic aspect, because it is in high coincidence with the end of transited supermillenial warming after the Wurm ice epoch (Imbree \& Imbree 1988). The magnitude of the quasy-bicenturial cycle is higher near the Maunder-type minima. The results concerning the Schove series are in a very good agreement with the aforesaid. 
A quasymillenial ( $T \sim 1000$ years) oscilation is most powerful in the magnitude behaviour of quasycenturial solar multiplet (70-130 years). Essentially weaker is the influence of 2200-2500-year cycle. Bonev, Penev \& Sello (2004) examined the last 4500 years of the ${ }^{14} C$ record, the continuous part of the Schove series (AD 296-1996), and the Group Sunspot Numbers. These authors applied two independent "local" methods for investigating unsteady non-linear time series: a "moving windows" periodogram algorithm and a multiresolution wavelet analysis. In their recent work Bonev, Penev \& Sello (2004) showed the changes related to the variability behavior of the quasi-century and two-century cycles on a very high scale-temporal and amplitude resolution (

The two parallel studies presented here propose several lines of evidence for an upcoming long-term solar variability minimum, which might be less deep than the Maunder minimum.

On the basis of the above mentioned analysis it can be concluded that the obtained results by use of different methods are in a very good agreement. The comparison between results based on tree ring radiocarbon data and Schove's series for the last $\sim 1700$ years are in strong coincidence too. The latter pointed out that the oldest reconstruction of large-scale structure of solar activity by D. J. Schove from AD 1955 and 1983 is not in contradiction, but rather in good agreement with the modern "cosmogenic" isothopes series. There is evidence for a significant change in the regime of centurial and supercenturial variations of solar activity during the Holocene. An essential part of this change is caused by modulation effects from quasibimillenial (2200-2500-year) and quasymillenial ( 900-1000-year) cycles.

About 5000-5500 BC a serious change in the regime of large-scale behaviour of solar activity has occurred. It is characterized by the establishment of the presnt structure of the 2200-2500-year cycle as well as by amplitude modulation effects of the same one over the shorter submillenial and quasycenturial solar cycles. The amplitude modulation effects of quasybimillenial and quasymillenial cycles over the shorter solar cycles is a very important feature of large-scale variations of solar activity. It is necessary to account for it if we would make a more precise solar activity forecasting model for time intervals in the range of few decades or more.

\section{References}

Benson, J. L., Bonev, B., James, P., Shan, K., Cantor, P. and M, C.: 2003, The Seasonal Behaviour of Water Ice Clouds in the Tharsis and Valles Marineris regions of Mars: Mars Orbiter Camera Observations, Icarus 165, 34-52.

Bonev, B., Penev, K. and Sello, S.: 2004, Long-term Solar Variability and the Solar Cycle in the XXI Century, Astrophys J. Lett pp. 81-84.

Hoyt, D. and Schatten, K.: 1998, Group Sunspot Numbers: A New Solar Activity Reconstruction, Solar Phys. 181(2), 491-512.

Imbree, J. and Imbree, K.: 1988, The Secret of Ice Epochs, russian edn, Mir, Moscow.

Komitov, B.: 1986, On a Possible Effect of Solar Cycles on the Climate of Bulgaria, number 5 in Solar Data, pp. 73-78.

Komitov, B.: 1997, The Schove's Series: Centurial and Supercenturial Variations of Solar Activity. Relationships between Adjacent Solar Cycles, Bulg. Geophys. J. 23(1-2), 69-79.

Komitov, B.: 1999, To Problems of Stability of the 100- and 200-Year Solar Cycles, Sixth National Conference on Sun-Earth Physics, Sofia, pp. 185-187.

Schove, D. J.: 1983, Sunspot Cycles, Stroudsburg: Hutchinson Ross.

Stuiver, M., Reimer, P. J., Bard, E., Beck, J. W., Burr, G. S., Hughen, K. A., Kromer, B., McCormac, F. G., v. d. Plicht, J. and Spurk, M.: 1998, Radiocarbon Age Calibration, 24,000-cal BP, INTCAL98, number 40 in Radiocarbon, pp. 1041-1083. 